\title{
HBV verdoppelt Mortalitätsrisiko von HIV
}

\author{
Mit Hepatitis-B-Virus koinfizierte \\ HIV-Patienten haben ein fast doppelt \\ so hohes Risiko für AIDS oder Tod als \\ mit HIV monoinfizierte Personen.
}

- Aufgrund gemeinsamer Risikofaktoren kommt eine HBV-Infektion bei HIV-infizierten wesentlich häufiger vor als in der Allgemeinbevölkerung. Derzeit herrscht die Ansicht vor, dass die HIV-Infektion einen ungünstigen Einfluss auf die Entwicklung von HBVassoziierten Leberschäden ausübt und mit höheren HBV-DNA-PolymeraseAktivitäten im Serum sowie einer höheren Raten an persistierendem HBe-Ag einhergeht.

Klinisch führen niedrige CD4-Zellzahlen zu einer erhöhten Rate von Leberzirrhose, leberbezogener Mortalität und hepatozellulärem Karzinom. Bei HIV-infizierten Patienten kommt es auch häufiger zu einem chronischen Verlauf der Hepatitis B.

Klinische Studien aus der Zeit vor der allgemeinen Verfügbarkeit der HAART erbrachten unterschiedliche Ergebnisse. Im Rahmen der laufenden US Military HIV Natural History Study, in der über $5200 \mathrm{HIV}$-infizierte Personen der amerikanischen Streitkräfte seit 1986 beobachtet werden, hat man den HBV-Status von 2352 HIV-Serokonvertern im Zeitraum von unter drei Jahren nach der Serokonversion untersucht und den weiteren Krankheitsverlauf verfolgt. 474 Personen (20\%) hatten eine durchgemachte Hepatitis B, 82 (3\%) wiesen isoliert anti-Hbc auf und 64
(3\%) hatten eine chronische Hepatitis B. Die nicht adjustierten Raten des Auftretens von AIDS bzw. Tod in diesen vier Gruppen betrugen 2,43, 3,27, 3,75 und 5,41. In einer multivarianten Analyse lag die Hazard Ratio für AIDS und Tod in der Gruppe der Patienten mit chronischer Hepatitis B im Vergleich zu HBV-negativen Personen bei 1,8. Personen mit durchgemachter Hepatitis B und isoliert vorhandenem anti-HBc wiesen dagegen nur nicht signifikant erhöhte Risiken auf.

\section{Kommentar}

Die Studie kann zwar einige interessante Hypothesen generieren, hinterlässt einen bezüglich des praktischen Vorgehens allerdings ratlos. Die Tatsache, dass es sich durchweg um Angehörige der Streitkräfte mit hervorragender medizinischer Versorgung handelt, macht die Übertragung der Untersuchung auf den Versorgungsalltag außerhalb der Armee kaum möglich. So finden sich in der Studie naturgemäß nur sehr wenige Frauen und praktisch keine Drogenabhängigen. Wie bei jeder Kohortenstudie, so lässt sich anhand der Daten über die möglichen Mechanismen, wie Hepatitis B den Krankheitsverlauf der HIV-Infektion beeinflusst, nur spekulieren. Bezüglich einer möglicherweise ebenfalls vorliegenden Hepatitis C-Infektion wurden die meisten Studienteilnehmer nicht suffizient untersucht.

H. S. FÜESSL :

\section{- H. M. Chun}

(Korres.: Helen M. Chun, MD, Department of Defense HIV/AIDS Prevention Program, Naval Health Research Center, 140 Sylvester Rd, San Diego CA 92106; helen.chun@med.navy.mil): Hepatitis B Virus Coinfection Negatively Impacts HIV Outcomes in HIV Seroconverters. J. Infect. Dis. 2012;205:185-193

\section{ART nach HIV-Serokonversion: früher oder später anfangen?}

\section{Der frühzeitige Beginn einer anti- retroviralen Therapie schiebt bei der frühen HIV-Infektion den Zeitpunkt etwas hinaus, zu dem die sicheren Kriterien für eine Therapieindikation auftreten.}

- Ob es für den Krankheitsverlauf günstig ist, wenn man die antiretrovirale Therapie (ART) möglichst frühzeitig im Verlauf der Infektion beginnt oder lieber die indizierende CD4-Zellzahlreduktion abwarten soll, ist nach wie vor unklar.

In einer multizentrischen Studie in den USA und in Peru hat man nun 150 Männer und Frauen über 18 Jahre mit einer im Lauf der letzten sechs Monate erworbenen HIV Infektion in eine frühzeitig behandelte und in eine klinisch indizierte Behandlungsgruppe randomisiert. Patienten der IT-Behandlungsgruppe erhielten eine festdosierte Kom- 